\title{
Executive summary: A step-by-step guide to strengthening sexual violence services in public health facilities
}

Jill Keesbury

Population Council

Jill Thompson

Follow this and additional works at: https://knowledgecommons.popcouncil.org/departments_sbsr-rh

Part of the Demography, Population, and Ecology Commons, Domestic and Intimate Partner Violence Commons, Family, Life Course, and Society Commons, Gender and Sexuality Commons, International Public Health Commons, Medicine and Health Commons, and the Women's Health Commons How does access to this work benefit you? Let us know!

\section{Recommended Citation}

Keesbury, Jill and Jill Thompson. 2010. "Executive summary: A step-by-step guide to strengthening sexual violence services in public health facilities." Lusaka: Population Council. 

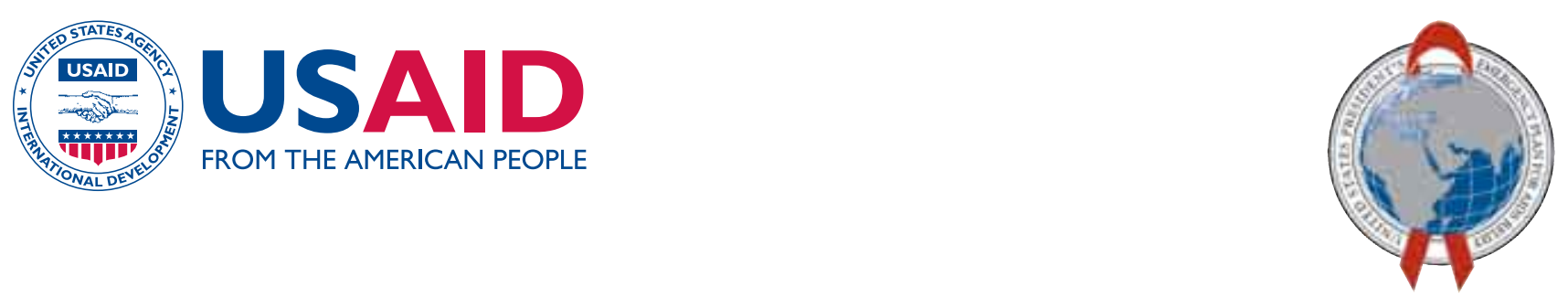

\section{EXECUTIVE SUMMARY}

\section{A Step-By-Step Guide to Strengthening Sexual Violence Services in Public Health Facilities}

Summary of "A step-by-step guide to strengthening sexual violence services in public health facilities: Lessons and tools from sexual violence services in Africa." Jill Keesbury and Jill Thompson, 2010, Lusaka: Population Council (available at www.popcouncil.org).

Sexual violence (SV) is a serious health and human rights problem across Africa that disproportionately affects women and girls. Survivors require comprehensive and sensitive care to mitigate the negative health consequences of SV, minimize psychological trauma and promote long-term reintegration and recovery. Despite high levels of sexual violence and a growing recognition of the need to improve the management of rape and sexual assault, many public health facilities in Africa do not currently have capacity to provide comprehensive, patient-centered SV care to adult and child survivors.

The purpose of this guide is to provide practical guidance on the steps necessary to establish and strengthen SV services within existing public health facilities, improve linkages to other sectors, and engage local communities around issues of sexual violence. It includes tips, resources and tools that will help partners and stakeholders design, implement and evaluate SV programs. The steps contained in the guide are summarized below.

\section{Step I: Collecting information needed to inform service strengthening and integration}

The first step in strengthening SV services is to learn as much as possible about how SV services are currently provided in health facilities, the attitudes, awareness and concerns of the community and service providers, other SV services and resources in the community and the linkages that exist between the health facility and other service providers, especially the police. Recommended methods for conducting such an assessment include:

- Conducting stakeholder consultations to identify needs and build a sense of ownership among key constituencies (facility management, police services, other government ministries, community leaders, NGOs and CBOs).

- Identifying the staff, equipment and services that are currently available at health facilities through a facility inventory.

- Holding focus group discussions with health care providers to assess knowledge and attitudes toward SV survivors and the challenges they face in providing comprehensive care.

- Identifying legal requirements for forensic evidence collection and documentation needed to support prosecution.

- Conducting community-based assessments to identify barriers and opportunities to accessing SV services, and promote community engagement in service strengthening. 


\section{Step 2: Establishing the regulatory and infrastructural framework for services}

Based on the findings of the assessment, program managers can begin to ensure that the regulatory and infrastructure frameworks are in place for delivering quality SV services. Essential components of these frameworks include:

- Facility-level policies and guidelines for management of SV, including referrals for services not available on-site.

- One location within the facility for providing all services in an integrated fashion, 24-hours a day, 7 days a week.

- Access to all necessary medicines, supplies and equipment at all times that SV services are provided.

- Adequate and appropriate record-keeping and reporting procedures, including medico-legal documentation for forensic evidence.

- Free or reduced-cost services for SV survivors.

\section{Step 3:Training health care providers}

Provider training is essential for ensuring access to quality SV services. As a multi-sectoral effort, some degree of training or sensitization is essential for all service providers, including health care workers, counsellors, police, paralegals and prosecutors.

- The initial training of health care providers can range from a one to two-day workshop to a two-week comprehensive training, depending on the needs identified at the intervention site and the availability of resources. At a minimum, all health care providers managing SV survivors should be qualified or trained in the following skills: provision of post-exposure prophylaxis for (PEP) for HIV, emergency contraception, basic counseling procedures, conducting and documenting forensic examinations, special considerations for children and adolescents, and making appropriate referrals.

- To the extent possible, it is recommended that the health facility identify and train dedicated practitioners (doctors, forensic nurses, and/or clinical officers) to conduct the medico-legal examinations in SV cases, particularly those involving young children.

- In addition to (or as part of) providing specialized training for health care providers, it is helpful to organize a shorter one to twoday multi-disciplinary orientation and training for all key local stakeholders involved in the management of rape and sexual assault cases.

\section{Step 4: Increasing PEP adherence and follow-up care}

Once SV and PEP services are introduced and routinely provided in the facility by trained providers, it is important to ensure ongoing care. Follow-up appointments are recommended to monitor the survivor's condition and treatment, assess her/his mental and emotional state, provide counseling and support for PEP adherence, and conduct follow-up tests for HIV. Nonetheless, many survivors do not access this care. Strategies for increasing adherence include:

- Minimizing the number of return visits required to obtain a complete course of PEP, ideally so that only one visit is required.

- Using the simplest regimen of PEP available and providing anti-emetics.

- Ensuring that follow-up arrangements and referrals are in place before the survivor leaves the facility. For children, these arrangements need to be organized through a parent or guardian.

\section{Step 5: Developing referral linkages to other service providers}

In most cases - even in "one-stop" settingsproviding comprehensive care to SV survivors will require one or more referrals from the primary care provider to other service providers located outside of (or inside) the health facility. Ensuring strong referral linkages to and from these providers is a 
critical, yet often underdeveloped element of clinical SV services. Referrals can be strengthened through:

- Identifying service providers in the community to whom and from whom SV survivors can be referred for services.

- Developing a formal referral directory and protocol for out-referrals, that is posted in the SV examination room.

- Develop a simple flow diagram which illustrates the key steps in the SV treatment protocol, and the order in which the steps take place.

- Developing monitoring systems to facilitate and track use of referral services.

\section{Step 6: Strengthening linkages between the health facility and communities to increase timely utilization of services}

To ensure the timely utilization of the services available within the health facility, it is critical that communities are aware that the services exist and how to access them. Essential messaging includes the need for survivors to seek SV health services as soon as possible, no later than 72 hours of the assault.

- Health facilities and their partners, especially the police, should adopt a multi-pronged approach to community awareness, combining information about services with messages to sensitize the community about the issue of SV. Multi-sectoral school outreach activities are especially effective for reaching children and adolescents.

- Posters, pamphlets, and other written materials on SV and SV services can be useful for conveying information and reinforcing messages, but must be culturally relevant and written in a language understood by community members.

- Messages on SV can be integrated into existing community outreach and health education activities, including health talks, as well as other routine services attended by women and girls such as reproductive, maternal and newborn health care.

\section{Step 7: Monitoring and evaluation}

Routine and systematic monitoring and evaluation (M\&E) of SV services is essential for ensuring that they quality and accessible to all survivors of SV. A good M\&E system also enables the identification of good practices and lessons that other health programs can learn from. At the minimum, M\&E systems supported by USG funds should employ the "PEPFAR Next Generation Indicators" to monitor the provision of PEP services; other indicators will be required to assess the overall quality and comprehensiveness of care provided.

Relevant PEPFAR Next Generation Indicators are :

- P6.I.D: Number of persons provided with PEP, by exposure type (occupational, rape/sexual assault victims, or other non-occupational)

- P6.2.N: Percentage of health facilities with HIV PEP available, by exposure type (occupational, non-occupational)

- PI2.2.D: Number of people reached by an individual, small group or community-level intervention or service that explicitly addressed gender-based violence and coercion related to HIVIAIDS

\section{Step 8: Ensuring adequate resources}

Planning for integrated SV services entails budgeting appropriately for start-up costs, developing a sustainability strategy for recurrent costs through establishing routine line items in annual budgets, and planning for scale-up from inception.

- The cost of establishing or strengthening SV services at an existing health facility will vary according to the setting, extent of training required, and level of equipment and services already available.

- Development partners should work with the facility management and other key stakeholders from the outset to develop a sustainability plan to support recurrent costs after the project to introduce and strengthen services ends. 
- It may also be possible to share costs with other organizations in the area working on SV issues or for partner organizations to take over responsibility for stakeholder coordination, outreach, and monitoring/evaluation over time.
- Scale-up of models for delivering integrated services should be planned and budgeted for at the outset of activities; all stakeholders, especially government, should be engaged and committed to scale-up before a pilot or demonstration project begins.

\section{Conclusion: Promoting country ownership, sustainability and scale-up}

Country ownership is essential for programmatic success, and the multi-sectoral nature of comprehensive SV services requires that a range of Ministries are included in program planning and implementation. In many countries this includes the Ministries of Health, Justice, Home Affairs (Police), Gender, and Community Services in addition to NGOs, the private sector, and civil society.

- While these stakeholders may work together at the community-level, they are less likely to collaborate centrally. To promote such coordination, many countries have formed national-level multi-sectoral working groups to guide the introduction and implementation of integrated packages of SV services.

- Within each participating Ministry, it is important to have designated persons or "focal points" to coordinate SV services at both national and district level.

- In some settings, sustainability can also be facilitated through forming partnerships with a strong, local health and/or social welfare NGO and/or through engaging private sector health providers.

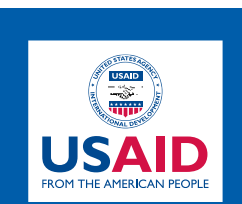

This report was made possible through support provided by the United States Agency for International Development (USAID) under the terms of Contract No. GHS-I-00-07-000 I I-00. The contents are the responsibility of the authors and do not necessarily reflect the views of USAID or the United States Government.

\section{Population Council}

The Population Council conducts research worldwide to improve policies, programs, and products in three areas: HIV and AIDS; poverty, gender, and youth; and reproductive health. www.popcouncil.org

Original report published in November 2010. Copyright @2010 United States Agency for International Development. 